\title{
Clinical trial protocol to evaluate the efficacy of cefixime in the treatment of early syphilis
}

Shivani N. Mehta ${ }^{1}$ (D) Chrysovalantis Stafylis ${ }^{1}$, David M. Tellalian², Pamela L. Burian², Cliff M. Okada ${ }^{2}$, Carl E. Millner², Christopher M. Mejia ${ }^{3}$ and Jeffrey D. Klausner ${ }^{1,2^{*}}$

\begin{abstract}
Background: Syphilis rates have been increasing both in the USA and internationally with incidence higher among men-who-have-sex-with-men and people living with human immunodeficiency virus (HIV) infection. Currently, benzathine penicillin is the recommended treatment for syphilis in all patients. Global shortages and cost increases in benzathine penicillin call for alternative treatment options. This study evaluates the efficacy of oral cefixime for the treatment of early syphilis.

Methods: We are conducting a randomized, multisite, open-label, non-comparative clinical trial in Los Angeles and Oakland, CA. Eligible participants are $\geq 18$ years old, with primary, secondary, or early latent syphilis (rapid plasma reagin [RPR] titer $\geq 1: 8$ ). Patients with HIV infection must have a viral load $\leq 200$ copies $/ \mathrm{mL}$ and CD4+ T cell count $\geq 350$ cells/ $\mu \mathrm{L}$ during the past 6 months. Participants are randomized to receive either $2.4 \mathrm{M}$ IU benzathine penicillin $\mathrm{G}$ intramuscularly once or cefixime $400 \mathrm{mg}$ orally twice a day for 10 days. Participants return at 3, 6, and 12 months post-treatment for follow-up RPR serological testing. The primary outcome is the proportion of participants who achieve $\geq 4$-fold RPR titer decrease at 3 or 6 months post-treatment.
\end{abstract}

Discussion: Clinical trials evaluating the efficacy of alternative antibiotics to penicillin are urgently needed.

(Continued on next page)

\footnotetext{
* Correspondence: JDKlausner@mednet.ucla.edu

'Department of Medicine, David Geffen School of Medicine at UCLA, Los

Angeles, CA, USA

${ }^{2}$ Department of Medicine, AIDS Healthcare Foundation, Los Angeles, CA, USA

Full list of author information is available at the end of the article
}

(c) The Author(s). 2020 Open Access This article is licensed under a Creative Commons Attribution 4.0 International License, which permits use, sharing, adaptation, distribution and reproduction in any medium or format, as long as you give appropriate credit to the original author(s) and the source, provide a link to the Creative Commons licence, and indicate if changes were made. The images or other third party material in this article are included in the article's Creative Commons licence, unless indicated otherwise in a credit line to the material. If material is not included in the article's Creative Commons licence and your intended use is not permitted by statutory regulation or exceeds the permitted use, you will need to obtain permission directly from the copyright holder. To view a copy of this licence, visit http://creativecommons.org/licenses/by/4.0/. The Creative Commons Public Domain Dedication waiver (http://creativecommons.org/publicdomain/zero/1.0/) applies to the data made available in this article, unless otherwise stated in a credit line to the data. 
(Continued from previous page)

Trial registration: Clinicaltrials.gov NCT03660488. Registered on 4 September 2018.

Keywords: Clinical trial, Cefixime, Treponema pallidum, Penicillin, Early syphilis, Syphilis

\section{Administrative information}

The order of the items has been modified to group similar items (see http://www.equator-network.org/reporting-guidelines/spirit-2013-statement-defining-standardprotocol-items-for-clinical-trials/).

\begin{tabular}{ll}
\hline Title $\{1\}$ & Clinical Trial Evaluating the Clinical \\
& Efficacy of Cefixime in Treatment for \\
& Early Syphilis \\
Trial registration $\{2 a$ and $2 b\}$. & ClinicalTrials.gov Identifier: \\
& NCT03660488 [1] \\
& All items from ClinicalTrials.gov registry \\
& can be directly found within the \\
& protocol. \\
& Version 9; 11.20 .2019 \\
Funding $\{4\}$ & AlDS Healthcare Foundation research \\
& grant (Grant \#20181796) \\
Author details $\{5 \mathrm{a}\}$ & 1. Department of Medicine, David \\
& Geffen School of Medicine at UCLA, Los \\
& Angeles, CA/United States of America \\
& 2. Department of Medicine, AIDS \\
& Healthcare Foundation, Los Angeles, \\
& CA/United States of America \\
& 3. Public Health Division, AIDS \\
Healthcare Foundation, Los Angeles, & CA/ United States of America \\
&
\end{tabular}

Name and contact information AIDS Healthcare Foundation for the trial sponsor $\{5 b\}$

Role of sponsor $\{5 c\}$

patients who are allergic to penicillin [5-8]. Injectable daily ceftriaxone is another alternative treatment that may be considered and is safe in pregnancy, as a recent review from our team showed [9].

Existing alternative treatment recommendations are based on clinical experience, a limited number of small clinical trials, and case series $[5-7,10]$. However, each regimen poses clinical challenges. Doxycycline/ tetracycline requires 14 days of treatment by mouth, with tetracycline requiring four daily doses. Ceftriaxone is administered intramuscularly, just like penicillin, but it requires daily injections for 10-14 days, making adherence potentially problematic. In pregnancy, only benzathine penicillin is recommended due to potential toxic effects of the alternatives or due to insufficient efficacy data (WHO). Shortages of benzathine penicillin worldwide have led to the use of unproven nonpenicillin alternatives [10-12].

Considering the high cost and time required for developing and approving new antibiotics that can treat syphilis in patients with and without HIV infection, a new approach for identifying new, safe, and efficacious antibiotic treatments for syphilis is necessary. Previously Food and Drug Administration (FDA)-approved antibiotics that are safe and efficacious in other infections and have a favorable pharmacologic profile suggesting activity against Treponema pallidum may be effective alternatives for treating syphilis.

Cefixime is an FDA-approved orally administered third-generation cephalosporin with spectrum of activity and pharmacokinetic profile similar to that of ceftriaxone, a drug which has been used for the treatment for syphilis [13]. Cefixime is clinically used for uncomplicated urinary tract infections, upper respiratory tract bacterial infections, and in the treatment of uncomplicated Neisseria gonorrhoeae genital infection [14]. To our knowledge, it has never been studied as a treatment for early syphilis.

Cefixime has a well-studied pharmacokinetic profile [14-19]. Unlike other alternatives for syphilis, adverse event profiles are favorable with cefixime in nonpregnant as well as pregnant patients [20, 21]. Nearly $40-50 \%$ of the dose is absorbed when it is given orally, whether administered with or without food. Peak concentrations occur between 2 and $6 \mathrm{~h}$ following oral administration of a single $400 \mathrm{mg}$ tablet. A single $400 \mathrm{mg}$ tablet produces an average peak concentration of approximately $3.7 \mu \mathrm{g} / \mathrm{mL}$ (range $1.3-7.7 \mu \mathrm{g} / \mathrm{mL}$ ). Typical available alternative treatments for non-pregnant

\section{Introduction}

Syphilis rates have been increasing both in the USA and $100,000(56,485$ reported cases) to 31.2 cases per 100 000 (101,584 reported cases). Nearly half of the new early cases of syphilis occur among men-who-have-sexwith-men, while incidence is also high among living with human immunodeficiency virus making syphilis an urgent global health threat [2Currently, benzathine penicillin is the recommended treatment for syphilis in all patients, including those liv- 
blood levels of cefixime after a single dose of cefixime $400 \mathrm{mg}$ by mouth are $4.84 \mu \mathrm{g} / \mathrm{mL}$ maximum at $4 \mathrm{~h}$ and above $1.0 \mu \mathrm{g} / \mathrm{mL}$ at $12 \mathrm{~h}$. Serum protein binding is concentration independent with a bound fraction of approximately $65 \%$. Cefixime is moderately distributed into extracellular water/tissue pools. Its half-life averages to $3-4 \mathrm{~h}$ but may range up to $9 \mathrm{~h}$ in healthy volunteers. Approximately $50 \%$ of the absorbed dose is excreted unmodified in the urine within $24 \mathrm{~h}$ and nearly $10 \%$ is excreted in bile [10].

We therefore believe that cefixime's pharmacokinetic similarity to ceftriaxone and its safety in the treatment of pregnant women could potentially make it a viable option in the treatment of early syphilis.

\section{Objective $\{7\}$}

The primary objective of our study is to determine the efficacy of cefixime $400 \mathrm{mg}$, taken orally two times a day (BID) for 10 consecutive days. Our hypothesis is that cefixime would be an efficacious treatment for early syphilis.

\section{Trial design $\{8\}$}

This is a randomized, open-label, non-comparative pilot clinical trial. Participants will be randomly assigned (1:1 allocation) to receive either the standard of care benzathine penicillin injection or 10-days of oral cefixime. The study will require 2.5 years to be completed and each participant will be part of the study for 1 year. This pilot study could set the foundation for a larger randomized clinical trial evaluating the clinical efficacy of oral cefixime versus benzathine penicillin for the treatment of early syphilis.

\section{Methods: Participants, interventions, and outcomes}

\section{Study setting $\{9\}$}

The study will take place in 3 primary care HIV healthcare clinics and 1 wellness center of the AIDS Healthcare Foundation in Los Angeles and Oakland, CA. Healthcare clinics offer HIV and sexually transmitted infection (STI) primary care services while wellness centers are walk-in comprehensive sexual health clinics that offer HIV/STI screening services, STI treatment, and other prevention services.

\section{Eligibility criteria $\{10\}$}

The inclusion criteria are:

1) Clinically or laboratory-confirmed new cases of early syphilis (primary, secondary, early latent syphilis) with a plasma rapid plasma reagin (RPR) $\geq 1: 8$

2) Eighteen years of age or older, capable of providing informed consent
3) HIV infected individuals must have CD4 T cell count $\geq 350$ cells $/ \mathrm{mm}^{3}$ and be virologically suppressed (viral load $<200$ copies $/ \mathrm{mL}$ ) during the past 6 months

4) Able to travel to clinic once a day or be available for phone calls or receive a text message for at least $7-10$ days

The exclusion criteria are:

1) Allergy to cefixime or penicillin

2) Pregnancy or a positive pregnancy test

3) Serofast RPR titer (prior titer $\geq 1: 8$ without a history of 4-fold titer decline)

4) Recent (within the past 7 days) or concomitant antimicrobial therapy with activity against syphilis, namely azithromycin, doxycycline, ceftriaxone, or other beta lactam antibiotics (e.g., amoxicillin)

5) A medical condition or other factors that might affect their ability to follow the protocol

\section{Who will take informed consent? $\{26 \mathrm{a}\}$}

Patients must provide written, informed consent before any study procedures occur (randomization, blood sample collection, treatment). Consent will be obtained by a study clinician in a private examination room. The clinician will explain the study goal, research procedures, participant rights, and obligations following the informed consent sheet. The participant will be able to ask questions and will be given adequate time to review the consent and decide if they wish to participate. After reading through the document, the participant will provide their signature if they would like to take part in the study.

\section{Additional consent provisions for collection and use of participant data and biological specimens $\{26 \mathrm{~b}\}$}

Patients will also sign a Health Insurance Portability and Accountability Act (HIPAA) release form allowing access to clinical and laboratory data, including their HIV test results.

\section{Interventions}

Explanation for the choice of comparators $\{6 \mathrm{~b}\}$

This is a pilot, non-comparative clinical trial designed to collect preliminary efficacy data. It includes an "experimental arm" of participants receiving cefixime and a contemporaneous "control arm" of participants receiving benzathine penicillin. The study was not designed to be adequately powered to show a statistically significant difference in the efficacy between the penicillin and cefixime arms. 


\section{Intervention description $\{11$ a}

Eligible participants who provide their consent are randomized to the two arms of the study. Initially, the study team collects demographic (age, gender, race, ethnicity, contact, sexual orientation) and clinical information (most recent RPR titer, CD4 T cell count, HIV viral load). A venipuncture blood sample is collected by trained clinic staff and it is sent to the laboratory for testing. Testing is conducted on serum using the Arlington scientific RPR test kit (Arlington, VA) [22]. Participants are randomly assigned to the two treatment groups. Participants assigned to the penicillin arm will receive 1 dose of $2.4 \mathrm{M}$ IU benzathine penicillin $G$ on the day of enrolment. Participants who are assigned to the cefixime arm will be given 20 capsules of oral cefixime $400 \mathrm{mg}$ on the day of enrolment to take for the following 10 days. Study staff observed receipt of the first dose. Subjects in the cefixime arm will then be asked to return for a clinical assessment or have a phone call assessment with the study team member 2 weeks following enrolment.

Study staff will follow up with all participants at 3, 6, and 12 months. In each follow-up, participants are asked questions regarding symptoms, antibiotic use in the past 3 months and the number of sex partners with whom they had condomless sex in the past 3 months. A new venipuncture blood sample is also collected for RPR testing.

\section{Criteria for discontinuing or modifying allocated interventions $\{11 \mathrm{~b}\}$}

Participants may request to leave the study or they may be withdrawn due to study-related adverse events. If a subject is discontinued from study participation due to an adverse event, they will be evaluated by the study clinicians for the need of additional treatment for syphilis. Safety data will be collected on any subject who is withdrawn from the study.

Participants in both study groups may receive additional treatment with penicillin, if they show no response to treatment (stable or absence of 4-fold decline at 6 months).

\section{Strategies to improve adherence to interventions $\{11 \mathrm{c}\}$}

To ensure retention of participants, follow-up visits will be scheduled to coincide with routine clinic appointments for HIV care or preventive sexual health appointments, which occur every 3 months. In addition, study staff will contact participants, either over the phone or via text message, before their scheduled follow-up appointment. Finally, participants will receive reimbursement for their time and transportation in the form of a gift card.
Relevant concomitant care permitted or prohibited during the trial $\{11 \mathrm{~d}\}$

Usual HIV care and treatment for the participant will continue throughout the trial. Concomitant antibiotic use during the participation in the study duration of the trial will be recorded.

\section{Provisions for post-trial care $\{30\}$}

Once participants complete the study, they will be able to continue receiving clinical care from the clinics. Participants study records will be reviewed and if necessary, additional treatment for syphilis will be administered according to the standard of care protocol.

\section{Outcomes $\{12\}$}

The primary outcome is the successful treatment of early syphilis by the 3- or 6-month follow-up. The participants' RPR titer will be used as the primary measure of outcome. Successful treatment is defined as an equal or greater than 4-fold RPR titer decrease, from baseline to 3 or 6 months after treatment.

\section{Participant timeline $\{13\}$}

Participants will be part of the study for 12 months. Study evaluations will occur at 3, 6, and 12 months post-treatment. See Fig. 1 for the participant timeline for the trial and Table 1 for the study assessments.

\section{Sample size $\{14\}$}

The primary outcome of the study will calculate the proportion of subjects with a 4-fold or greater decrease in RPR titer from baseline at 3 or 6 months in the per protocol analysis population.

Assuming a treatment success ratio of $90 \%$ among participants receiving cefixime, a sample size of 40 participants will yield a 95\% confidence interval of (7697\%) (Table 2). Assuming 20\% attrition due to loss to follow-up or non-compliance with the study medication schedule, enrolling 50 subjects will provide 40 evaluable subjects in the PP analysis population. Similarly, we will enroll 50 participants into the penicillin arm, as a contemporary cohort.

\section{Recruitment $\{15\}$}

Participant recruitment will occur in 4 AIDS Healthcare Foundation (AHF) Clinics based in Los Angeles, CA, and Oakland, CA. During the scheduled clinical visit, the study clinicians will approach cases of syphilis returning for treatment and they will provide a brief overview of the study. If the participant is interested, the clinician will determine patient eligibility. If the patient is eligible and agrees to participate, the clinician will conduct the informed 


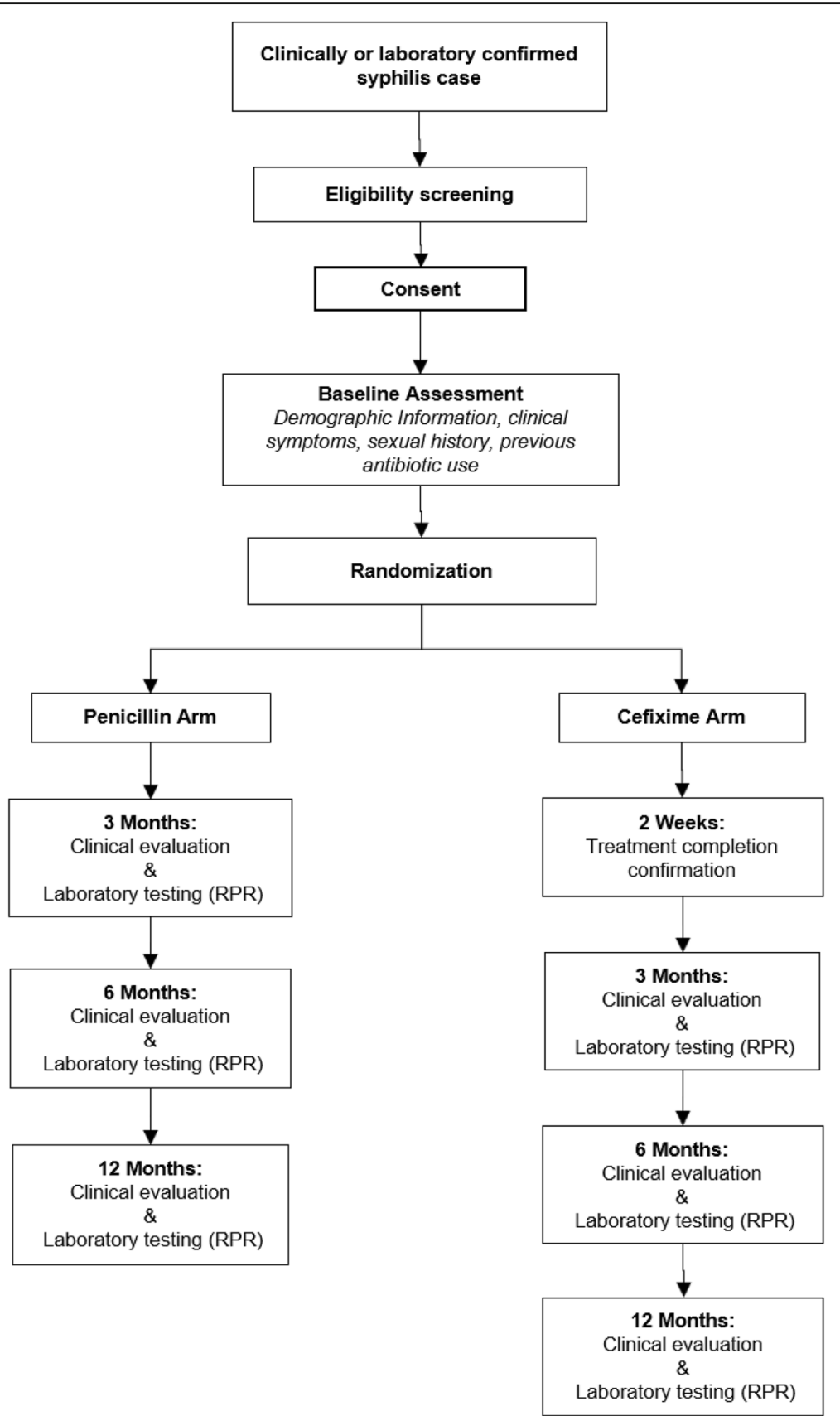

Fig. 1 Participant enrolment and follow-up schedule

consent and enrolment. Participants will be able to discuss study details with the clinicians and ask questions before signing the informed consent. Following enrolment, participants will be randomized, do their laboratory test, and receive the assigned treatment. Enrolment will be conducted by clinicians, who will be trained on the study procedures, and research assistants, who will assist in data collection, form preparation.

\section{Assignment of interventions: Randomization} Sequence generation $\{16 \mathrm{a}\}$

After consent, participants are randomly assigned to the study arms with a 1:1 allocation. We will use a simple randomization method with a shuffled deck of sealed envelopes that contain a card with the assigned treatment. One hundred randomization cards, 50 for each study group, will be created before the initiation of enrolment, sealed in unmarked envelopes, and they will 
Table 1 Study assessments by study time point

\begin{tabular}{|c|c|c|c|c|c|}
\hline \multirow[t]{2}{*}{ Assessment } & \multicolumn{5}{|l|}{ Time point } \\
\hline & Enrolment & 10-day follow-up (cefixime arm only) & 3-month visit & 6-month visit & 12-month visit \\
\hline Eligibility screening & $x$ & & & & \\
\hline Consent & $x$ & & & & \\
\hline Randomization & $x$ & & & & \\
\hline Demographic information & $x$ & & & & \\
\hline Clinical \& laboratory information & $x$ & & & & \\
\hline Blood sample collection (RPR and RPR titer) & $x$ & & $x$ & $x$ & $x$ \\
\hline Treatment administration & $x$ & & & & \\
\hline Evaluation of treatment completion & & $x$ & & & \\
\hline Recent sexual history & $x$ & $x$ & $x$ & $x$ & $x$ \\
\hline Recent antibiotic use & $x$ & $x$ & $x$ & $x$ & $x$ \\
\hline
\end{tabular}

be distributed randomly to each of the study sites. No other factors will be taken into consideration for randomization.

\section{Concealment mechanism $\{16 \mathrm{~b}\}$}

The envelopes containing the randomization cards are sealed; thus, the team member conducting enrolment does not know the content of the envelope.

\section{Implementation $\{16 c\}$}

The study staff will ask the participant to select a sealed envelope from the shuffled deck. After selecting the envelope, the participant will reveal the treatment to themselves and the study staff.

\section{Assignment of interventions: Blinding} Who will be blinded $\{17 \mathrm{a}\}$

This is an open-label clinical trial, and thus, neither the participants nor the study staff will be blinded to the assigned treatment. The data analyst performing interim and final statistical analyses will be masked to treatment assignment.

\section{Procedure for unblinding if needed $\{17 b\}$}

Not applicable.

Table 2 Sample size and 95\% confidence interval for successful treatment proportion of $90 \%$ and $95 \%$ among participants of the cefixime or penicillin arm

\begin{tabular}{lll}
\hline $\begin{array}{l}\text { Sample size of } \\
\text { evaluable subjects }\end{array}$ & $\begin{array}{l}\text { Efficacy proportion } \\
\text { of } \mathbf{9 0 \%}\end{array}$ & $\begin{array}{l}\text { Efficacy proportion } \\
\text { of } \mathbf{9 5 \%}\end{array}$ \\
\hline 20 & $68-99 \%$ & $0.75,1.0$ \\
40 & $76-97 \%$ & $0.83,0.99$ \\
60 & $79-96 \%$ & $86-99 \%$
\end{tabular}

$95 \%$ confidence intervals calculated using a binomial exact test

\section{Data collection and management}

Plans for assessment and collection of outcomes $\{18 \mathrm{a}\}$

A venipuncture blood sample will be collected at 3-, 6-, and 12-month visits, and it will be tested for RPR titer. Study data collected on baseline include basic demographic information, sexual history, and laboratory tests (CD4 count, viral load, RPR titer). On each follow-up visit, sexual history, antibiotic use, symptoms, and the RPR titer will be collected. Data will be collected on paper data collection forms and will be entered into Research Electronic Data Capture (REDCap) [23, 24]. The paper data collection forms were specifically designed for our study by the study research team to capture data relevant to our objective. Data quality and completeness are ensured through a weekly internal review process in which the research assistants review all data collection forms for completeness and the database for missing information.

\section{Plans to promote participant retention and complete follow-up $\{18 b\}$}

Study follow-up visits are scheduled to coincide with routine clinic appointments within AHF. Study staff will send participants a 1-month, 2-week, and 1-day notification prior to their follow-up appointment.

\section{Data management $\{19\}$}

Participant data will be collected on paper data collection forms and entered into REDCap. Data that will be entered into REDCap include participant information (name, date of birth, medical record number, contact information) and laboratory results.

\section{Confidentiality $\{27\}$}

REDCap servers are encrypted, HIPAA-compliant, password-protected, and accessible only by designated study members. Hard copy data collection forms will be 
stored into a locked cabinet with limited access only to designated members.

Plans for collection, laboratory evaluation, and storage of biological specimens for genetic or molecular analysis in this trial/future use $\{33\}$

Participants will provide a serum sample on baseline and at each follow-up visit. Samples will be collected by trained laboratory personnel inside the clinics. Samples will be sent to the laboratory within $24 \mathrm{~h}$ of collection. Sample processing and analysis will be conducted by trained laboratory staff following standard laboratory procedures. Samples will be destroyed after serological testing. The study team will not be involved in the sample collection, processing, analysis, and reporting.

There are no plans in this trial to evaluate or store biological specimens for genetic or molecular analysis for future use.

\section{Statistical methods}

Statistical methods for primary and secondary outcomes $\{20 \mathrm{a}\}$

The primary analysis for the main outcome will be conducted on the "per protocol" (PP) population. This will include participants who satisfy the inclusion and exclusion criteria, completed treatment (i.e., received the penicillin injection or received all of the cefixime pills), report no adverse events, returned for follow-up visits ( 3 and/or 6 months), and have an evaluable RPR result.

For each treatment group, we will calculate the proportion of PP participants who achieved a 4-fold RPR titer decrease at 3 or 6 months post-treatment ("treatment success") and the exact binomial 95\% confidence interval. Qualitative variables will be presented as frequencies with percentages and 95\% confidence intervals (95\% CIs) and quantitative variables as mean with standard deviation and range.

\section{Methods for additional analyses (e.g., subgroup analyses) \{20b\}}

There will be no other additional analyses beyond the main analyses for the primary and secondary outcomes.

\section{Methods in analysis to handle protocol non-adherence} and any statistical methods to handle missing data $\{20 \mathrm{c}\}$ Participants who do not adherence to the protocol or participants with missing data will not be included in the primary outcome analysis. We will be conducting an "intention to treat" (ITT) analysis that will include all individuals enrolled in the study, with evaluable RPR titter results and regardless of protocol non-compliance. For this analysis, missing data and non-adherent cases will also be considered as "treatment failure." Similarly to the PP analysis, we will calculate the treatment success proportion for each treatment group among the ITT population at 3 , or 6 months post-treatment and the exact binomial 95\% confidence interval.

\section{Interim analyses $\{21 \mathrm{~b}\}$}

A summary of the enrolment progress, treatment success proportions, adverse events, and protocol deviations will be provided to the Data Safety Monitoring Board members.

Plans to give access to the full protocol, participant leveldata, and statistical code $\{31 \mathrm{c}\}$

The protocol of the study is publicly available on ClinicalTrials.gov (NCT03660488). Deidentified data will be available upon request to the study Primary Investigator.

\section{Oversight and monitoring}

Composition of the coordinating center and trial steering committee $\{5 \mathrm{~d}\}$

The immediate study research team based in the University of California of Los Angeles meets on a weekly basis and oversees study recruitment, data quality, and study staff trainings. The immediate team is joined by a wider team of AHF study clinicians, based in study clinics, who also meet on a weekly basis.

Composition of the data monitoring committee, its role, and reporting structure $\{21 \mathrm{a}\}$

The Data and Safety Monitoring Board (DSMB) will be composed of a physician, biostatistician, and regulatory affairs specialist/ethicist and will oversee the study throughout the 2-year study period. They will review study activities every 6 months. The committee will review safety data and clinical efficacy reports and determine whether it is clinically safe to continue the clinical trial. They will report their recommendation to the Primary investigator.

\section{Adverse event reporting and harms $\{22\}$}

Cefixime and penicillin have a known safety and adverse event profile. Participants receiving penicillin may experience temporary mild pain on the site of injection. Cefixime can cause mild gastrointestinal reactions, such as diarrhea, loose stools, abdominal pain, and nausea [14]. Mild to severe allergic reactions are also expected in persons allergic to penicillin or cefixime.

Adverse events will be collected by spontaneous selfreport. Study participants will be asked to report any problems throughout their participation to the study and specifically the duration of the treatment. We will use the Division of AIDS (DAIDS) Adverse Event grading system [https://rsc.niaid.nih.gov/clinical-researchsites/daids-adverse-event-grading-tables] that classifies 
adverse events by organ system and severity. Any AE/ SAEs will be reported descriptively on the final project report and future publications.

The study site investigators will report serious adverse events and adverse events to the responsible IRB for that study site in accordance with respective IRB policies and procedures. Follow-up information to a reported adverse event will be submitted to the IRB as soon as the relevant information is available.

\section{Frequency and plans for auditing trial conduct $\{23\}$}

The trial and individual clinic sites will be audited at least once during the duration of the study by the study sponsor (AIDS Healthcare Foundation).

\section{Plans for communicating important protocol amendments to relevant parties (e.g., trial participants, ethical committees) $\{25\}$}

Amendments will be submitted to the IRB according to policies and guidelines. Any protocol changes will be promptly communicated to the IRB, the DSMB, and the study team.

\section{Dissemination plans $\{31 \mathrm{a}\}$}

We plan to disseminate study results through peerreviewed journal publications and conference presentations. Study findings will also be shared with relevant clinical and scientific groups.

\section{Discussion}

This is a randomized, non-comparative, pilot study evaluating the efficacy of daily oral cefixime $400 \mathrm{mg}$ for 10 days for the treatment of early syphilis. As syphilis rates increase and penicillin shortages continue to occur in the USA and worldwide, alternative treatments that are efficacious for both pregnant and non-pregnant populations, regardless of HIV status, are needed [6-8]. Already approved and antibiotics in clinical use with favorable pharmacokinetic profile, such as cefixime, should be clinically evaluated for alternative treatment options.

Data from this pilot study could be used as a foundation to assess the clinical effectiveness for cefixime in early syphilis treatment. Currently, our study is being conducted among non-pregnant individuals. However, subsequent clinical studies should also include women and pregnant women to address the gap in the treatment of maternal syphilis.

\section{Trial status}

Recruitment was initiated on September 16, 2018, and will be complete on January 15, 2021. The current protocol version is version $9(11 / 20 / 2020)$.
Authors' contributions $\{31 \mathrm{~b}\}$

SM led the writing of the manuscript. CS led the proposal and protocol development and contributed to the writing of the manuscript. DT, PB, CO, and CM contributed to clinical and logistical aspects of protocol development. JDK led the proposal and protocol development and developed the treatment algorithm for the protocol. All named authors adhere to the authorship guidelines of Trials. All authors have approved the final manuscript and agreed to publication.

The database for this study was supported by NIH National Center for Advancing Translational Science (NCATS) UCLA CTSI Grant Number UL1TR001881.

\section{Competing interests $\{\mathbf{2 8}\}$}

The authors declare that they have no competing interests.

\section{Funding $\{4\}$}

The trial is funded by AHF (Grant \#20181796). The funding body had no role in the design of the study, collection of data, or the writing of this paper, nor will the funding body have a role in analysis, interpretation of data, or in writing future manuscripts.

Availability of data and materials $\{29\}$

The datasets analyzed during the current study are available from the corresponding author on reasonable request.

Consent for publication $\{32\}$

Not applicable

Ethics approval and consent to participate $\{24\}$

The study was approved by Western IRB (IRB\# 20181796) and UCLA IRB (IRB\# 18-000665). Informed consent will be obtained from all study participants.

\section{Author details}

'Department of Medicine, David Geffen School of Medicine at UCLA, Los Angeles, CA, USA. '2Department of Medicine, AIDS Healthcare Foundation, Los Angeles, CA, USA. ${ }^{3}$ Public Health Division, AIDS Healthcare Foundation, Los Angeles, CA, USA.

Received: 6 May 2020 Accepted: 10 November 2020

Published online: 09 December 2020

References

1. ClinicalTrials.gov. Cefixime for alternative syphilis treatment. Bethesda: National Library of Medicine (US); 2018. Identifier NCT03660488. Available from: https://clinicaltrials.gov/ct2/show/NCT03660488. Cited 2020 April 29.

2. Braxton J, Grey J, Harvey A, Kidd S, Presley RJ, Ramirez V, et al. Syphilis surveillance supplement 2013-2017 for sexually transmitted disease surveillance 2017. 2019.

3. Kojima N, Klausner JD. An update on the global epidemiology of syphilis. Curr Epidemiol Rep. 2018;5(1):24-38.

4. World Health Organization. WHO guidelines for the treatment of Treponema pallidum (syphilis). 2016.

5. Clement ME, Okeke NL, Hicks CB. Treatment of syphilis: a systematic review. JAMA. 2014;312(18):1905-17.

6. Ghanem KG, Erbelding EJ, Cheng WW, Rompalo AM. Doxycycline compared with benzathine penicillin for the treatment of early syphilis. Clin Infect Dis. 2006;42(6):e45-e9.

7. Wong $T$, Singh AE, De P. Primary syphilis: serological treatment response to doxycycline/tetracycline versus benzathine penicillin. Am J Med. 2008; 121(10):903-8

8. Centers for Disease Control and Prevention. 2015 STD Treatment Guidelines. 2015. Available from: https://www.cdc.gov/std/tg2015/default.htm.

9. Roberts CP, Raich A, Stafylis C, Klausner JD. Alternative treatments for syphilis during pregnancy. Sex Transm Dis. 2019;46(10):637-40.

10. Nurse-Findlay S, Taylor MM, Savage M, Mello MB, Saliyou S, Lavayen M, et al. Shortages of benzathine penicillin for prevention of mother-to-child transmission of syphilis: an evaluation from multi-country surveys and stakeholder interviews. PLoS Med. 2017;14(12):e1002473.

11. Taylor MM, Zhang X, Nurse-Findlay S, Hedman L, Kiarie J. The amount of penicillin needed to prevent mother-to-child transmission of syphilis. Bull World Health Organ. 2016;94(8):559. 
12. World Health Organization. Shortages of benzathine penicillin. How big is the problem? And why it matters. 2017 [updated 2017, 27 December]. Available from: https://www.who.int/reproductivehealth/shortagesbenzathine-penicillin/en/.

13. Liang Z, Chen Y-P, Yang C-S, Guo W, Jiang X-X, Xu X-F, et al. Meta-analysis of ceftriaxone compared with penicillin for the treatment of syphilis. Int J Antimicrob Agents. 2016;47(1):6-11.

14. Suprax (cefixime) Tablets USP, 400 mg; [package insert]. Baltimore: Lupin Pharmaceuticals, Inc; 2012

15. Faulkner R, Bohaychuk W, Haynes J, Desjardins R, Yacobi A, Silber BM. The pharmacokinetics of cefixime in the fasted and fed state. Eur I Clin Pharmacol. 1988;34(5):525-8.

16. Faulkner R, Fernandez P, Lawrence G, Sia L, Falkowski A, Weiss A, et al. Absolute bioavailability of cefixime in man. J Clin Pharmacol. 1988;28(8): 700-6.

17. Nix DE, Symonds WT, Hyatt JM, Wilton JH, Teal MA, Reidenberg P, et al. Comparative pharmacokinetics of oral ceftibuten, cefixime, cefaclor, and cefuroxime axetil in healthy volunteers. Pharmacotherapy. 1997;17(1):121-5.

18. Schatz BS, Karavokiros KT, Taeubel MA, Itokazu GS. Comparison of cefprozil, cefpodoxime proxetil, loracarbef, cefixime, and ceftibuten. Ann Pharmacother. 1996;30(3):258-68.

19. Zakeri-Milani P, Valizadeh $H$, Islambulchilar Z. Comparative bioavailability study of two cefixime formulations administered orally in healthy male volunteers. Arzneimittel-Forschung. 2008:58(2):97-100.

20. Barbee LA, Nayak SU, Blumer JL, O'Riordan MA, Gray W, Zenilman JM, et al. A Phase 1 pharmacokinetic and safety study of extended-duration, highdose cefixime for cephalosporin-resistant Neisseria gonorrhoeae in the pharynx. Sex Transm Dis. 2018:45(10):677-83.

21. Rafal'skii W, Kozyrev I, Gustovarova TA, Khlybova SV, Novoselova AV, Filippenko NG, Likhikh DG. The efficacy and safety of cefixime and amoxicillin/clavulanate in the treatment of asymptomatic bacteriuria in pregnant women: a randomized, prospective, multicenter study. Urologiia (Moscow, Russia: 1999). 2013(5):24-6.

22. Arlington Scientific Inc. RPR card test for syphilis. 2006.

23. Harris PA, Taylor R, Thielke R, Payne J, Gonzalez N, Conde JG. Research electronic data capture (REDCap) - a metadata-driven methodology and workflow process for providing translational research informatics support. J Biomed Inform. 2009;42(2):377-81.

24. Harris PA, Taylor R, Minor BL, Elliott V, Fernandez M, O'Neal L, et al. The REDCap consortium: building an international community of software platform partners. J Biomed Inform. 2019;95:103208.

\section{Publisher's Note}

Springer Nature remains neutral with regard to jurisdictional claims in published maps and institutional affiliations.

Ready to submit your research? Choose BMC and benefit from:

- fast, convenient online submission

- thorough peer review by experienced researchers in your field

- rapid publication on acceptance

- support for research data, including large and complex data types

- gold Open Access which fosters wider collaboration and increased citations

- maximum visibility for your research: over $100 \mathrm{M}$ website views per year

At $\mathrm{BMC}$, research is always in progress.

Learn more biomedcentral.com/submissions 